\title{
Liquid-Phase Peak Force Infrared Microscopy for Chemical Nano- imaging and Spectroscopy of Soft Matters
}

\author{
Haomin Wang, ${ }^{1}$ Joseph M. González-Fialkowski, ${ }^{1}$ Wenqian Li, ${ }^{2}$ Qing Xie, ${ }^{1}$ Yan Yu, ${ }^{2}$ and Xiaoji \\ G. $\mathrm{Xu}^{* 1}$ \\ ${ }^{1}$ Department of Chemistry, Lehigh University, 6 E Packer Ave., Bethlehem, PA, 18015, United \\ States \\ ${ }^{2}$ Department of Chemistry, Indiana University, 800 E Kirkwood Ave, Bloomington, IN, 47405, \\ United States \\ *E-mail: xgx214@lehigh.edu
}

\begin{abstract}
Peak force infrared (PFIR) microscopy is an emerging atomic force microscopy that bypasses Abbe's diffraction limit in achieving chemical nano-imaging and spectroscopy. The PFIR microscopy mechanically detects the infrared photothermal responses in the dynamic tip-sample contact of peak force tapping mode, and has been applied for a variety of samples, ranging from soft matters, photovoltaics heterojunctions, to polaritonic materials under the air conditions. In this article, we develop and demonstrate the PFIR microscopy in the liquid phase for soft matters and biological samples. With the capability of controlling fluid compositions on demand, the liquid-phase peak force infrared (LiPFIR) microscopy enables in situ tracking the polymer surface reorganization in fluids and detecting the product of click chemical reaction in the aqueous phase. Both broadband spectroscopy and infrared imaging with $10 \mathrm{~nm}$ spatial resolution are benchmarked in the fluid phase, together with complementary mechanical information. We also demonstrate the LiPFIR microscopy on revealing the chemical composition of a budding site of yeast cell wall particles in water as an application on biological structures. The label-free, non-destructive chemical nano-imaging and spectroscopic capabilities of the
\end{abstract}


LiPFIR microscopy will facilitate the investigations of soft matters and their transformations at the solid/liquid interface. 


\section{Introduction}

While Abbe's diffraction limit restricts the spatial resolution of traditional infrared (IR) microscopy to half of the wavelength of several microns ${ }^{1}$, sub-micron resolutions have been achieved in several super-resolution IR microscopy methods. For instance, in depth-resolving mid-infrared photothermal (MIP) microscopy, micromolar sensitivity and a spatial resolution of $600 \mathrm{~nm}$ were reported. ${ }^{2}$ In ultraviolet-localized mid-infrared photoacoustic microscopy (ULMPAM), high-throughput and water background-free images of cultured cells can be obtained with a diffraction-limited resolution of $250 \mathrm{~nm} .{ }^{3}$ However, the spatial resolution of these techniques is still diffraction-limited by the shortest wavelength involved in excitation or signal detection, which cannot be easily aligned down to $<100 \mathrm{~nm}$ due to the limitation of the numerical aperture of IR objectives.

Integration of atomic force microscopy (AFM) with light radiation creates routes to achieve nanometer-scale spatial resolution far below the diffraction limit, ${ }^{4,5}$ thanks to the highly concentrated light field formed around the apex of the metallic tip. For example, vibrational imaging and Raman spectroscopy of single molecules have been achieved by the state-of-the-art tip-enhanced Raman scattering (TERS) with plasmonic enhancement. ${ }^{6-9}$ For IR microscopy, current AFM-based IR spectroscopic imaging methods can be separated into two categories based on different detection schemes: optical detection through the elastic light scattering from the optical nearfield of the metallic tip;, ${ }^{50}$ and mechanical detection of IR-induced actions between the sharp AFM tip and the sample as a result of light-matter interactions. The mechanical detection approach is particularly suitable for vibrational imaging and spectroscopy of soft matters, with high sensitivity and simple instrument design. ${ }^{11-13}$ Photothermal induced resonance (PTIR), ${ }^{14,}{ }^{15}$ resonantly enhanced infrared photo-expansion nanoscopy (REINS), ${ }^{16}$ 
photo-induced force microscopy (PiFM), ${ }^{12}$ and peak force infrared (PFIR) microscopy ${ }^{13}$ are increasingly popular representatives of AFM-based mechanical-detection IR methods, detecting IR signals directly proportional to sample's absorption profile. PTIR and REINS are based on the detection of the photothermal response from IR absorptions in the AFM contact mode operation. ${ }^{11,16}$ In contrast, PiFM, operating in the AFM tapping mode, detects the photo-induced force either of dipole-dipole origin,${ }^{17}$ optical-mechanical damping origin, ${ }^{18}$ or photothermal expansions through modified van der Waals force. ${ }^{19}{ }^{20}$ The PFIR microscopy, which operates with the peak force tapping mode (also known as the pulsed force mode), ${ }^{21}$ combines the respective advantages of PTIR and PiFM. It achieves a high spatial resolution of $<10 \mathrm{~nm}$ and non-destructiveness to the sample surface like PiFM, and unambiguous photothermal signal generation mechanism like PTIR/REINS. The PFIR microscopy also offers complimentary mechanical information channels, such as modulus and adhesion on the sample surface, allowing for simultaneous multimodal imaging together with the IR absorption. ${ }^{13,22}$

On the flip side, a typical obstacle for IR microscopies is the lack of straightforward compatibility with aqueous conditions. Water strongly attenuates the IR radiation, making the delivery and detection of IR in traditional far-field microscopy extremely challenging. The utilization of total internal reflection could reduce the delivery loss of IR radiation. However, the spatial resolution of attenuated total internal reflection Fourier transform infrared (ATR-FTIR) microscopy is bound by the optical diffraction limit to several microns. ${ }^{23}$ The AFM-based IR microscopies can address this challenge. ${ }^{22,}{ }^{24-29}$ With the total internal reflection geometry, evanescent IR field locally excites the vibrational modes of molecules and materials; the AFM tip probes the actions of the photothermal response excited by the evanescent field, avoiding the attenuation loss of the optical detection. REINS and contact mode PTIR have been demonstrated 
in the fluid phase on polymers and protein samples with a $20 \sim 25 \mathrm{~nm}$ spatial resolution. ${ }^{24,25} \mathrm{In}$ addition, scattering-type scanning near-field optical microscopy (s-SNOM) with total internal reflection and bottom-up illumination have also been applied in water. ${ }^{26,27,29} \mathrm{~s}$-SNOM uses the optical detection scheme and is based on the tapping mode AFM that avoids scratching the sample surface by the tip. However, the optical detection scheme of the s-SNOM suffers from an additional loss from water absorption after the near-field scattering by the metallic tip. Moreover, the s-SNOM apparatus has a higher instrumental complexity and often requires two separate types of light sources for IR imaging and spectroscopy. Recently, PiFM has also been implemented in the liquid, ${ }^{30}$ however, the high hydraulic drag of the fluid damps the mechanical oscillations of the cantilever, reducing the efficiency for the heterodyne force detection scheme. ${ }^{31}$

Peak force tapping mode has been proven to operate stably in the liquid phase without sample damage for living cells ${ }^{32}$ and single membrane proteins. ${ }^{33}$ High-resolution AFM images of peak force tapping mode are routinely achieved in the liquid phase. PFIR microscopy inherits those advantages of peak force tapping for operations in liquids. Our group has developed liquidphase peak force infrared (LiPFIR) microscopy by combining the photothermal detection mechanism of PFIR with the total internal reflection field delivery scheme. Imaging of phonon polaritons in hexagonal boron nitride submerged in water was demonstrated. ${ }^{28}$ In this article, we further develop the LiPFIR microscopy with the ability to adjust the types of fluids on-demand to initiate physical transformations and chemical reactions. We demonstrate that LiPFIR is generally suitable for label-free non-destructive chemical imaging of soft matters. We also quantify its spatial resolution to be $\sim 10 \mathrm{~nm}$. IR imaging and spectroscopy on polymer surface reorganization and click chemistry are demonstrated in situ. Finally, a biological sample of two 
zymosan particles in the budding process is imaged in water, revealing the presence of proteins around the budding site.

\section{Results and Discussion}

\section{Operational Principle and Design of LiPFIR Microscopy}

LiPFIR microscopy inherits the operational principle of PFIR microscopy ${ }^{13}$ with the addition of evanescent field delivery. It uses an AFM cantilever to mechanically detect photothermal expansions of the sample submerged in the liquid. The challenge of high attenuation of IR in water is overcome by the utilization of the total internal reflection geometry with a germanium prism. The photothermal signal of the IR absorption is mechanically detected in LiPFIR, so the would-be propagation loss of the IR signal in the optical detection after the light-matter interaction is bypassed. Figure 1a illustrates the total internal reflection geometry used in the LiPFIR microscopy. An AFM tip (we mostly use gold-coated tips) is placed above the germanium surface, and IR pulses are guided from the bottom at an incident angle of $20^{\circ}$ to enable the total internal reflection. A finite-difference time-domain (FDTD) simulation of such a scheme is displayed in Figure 1b, where an intensity enhancement of $5.2 \times 10^{2}$ is achieved at the germanium surface underneath the AFM tip. Spatial confinement with the full width at half maximum (FWHM) of only $25 \mathrm{~nm}$ is observed, which is vital for AFM-based super-resolution imaging. This tip-enhanced field excites local sample molecules. The absorbed energy is then converted into photothermal expansions, which are mechanically detected by the motion of AFM cantilever. Peak force tapping (PFT) mode is used in LiPFIR. In PFT mode, the distance between the tip and sample oscillates at a low frequency of a few $\mathrm{kHz}$. The tip momentarily contacts the sample surface for tens of microseconds and detaches afterward in every PFT cycle. ${ }^{21,34}$ The 
shear force that is built up in the lateral scan is released when the tip and the sample detach, avoiding the stick-slip motion of the contact mode AFM. ${ }^{35}$ Unlike tapping mode AFM, the feedback of PFT is based on the maximal cantilever deflection during the tip-sample contact, which is not affected by the hydrodynamic drag force of the fluid.

Figure 1c illustrates the LiPFIR apparatus consisting of a frequency tunable IR source, a liquid-phase compatible AFM, and a germanium prism. A quantum cascade laser (QCL, MIRcatQT, DRS Daylight Solutions) provides tunable IR laser pulses up to $1 \mu$ s duration with adjustable repetition rates. The beam from QCL is firstly expanded by a reflective telescope (BE04, Thorlabs) and then coupled into a germanium prism using a germanium lens with $2.5-\mathrm{cm}$ focal length. A PFT-enabled AFM (Bioscope Catalyst, Bruker) operates on the germanium prism with a metal-coated probe and the PFT frequency of $1 \mathrm{kHz}$. A transistor to transistor logic (TTL) waveform from the phase lock loop (PLL) at the PFT frequency is routed to a function generator in the burst mode to trigger the QCL to emit a train of IR pulses. The timing of the emission is set to the moment when the tip and sample are in dynamic contact. The deflection of the cantilever is read out by a built-in quadrant photodiode in AFM. A perfusion chamber is sealed by an O-ring to enclose both the cantilever and the sample. The perfusion chamber has a fluid inlet and an outlet, enabling changing the fluid composition to initiate physical transformations and chemical reactions.

The calculated relationship between the Z-component of the evanescent field $E_{z}$ and the incident angle $\theta$ is plotted in Figure $1 \mathrm{~d} \cdot{ }^{36}$ As the incident angle approaches the critical angle $\theta_{c}$ (which is calculated to be $19^{\circ}$ ), $\mathrm{E}_{\mathrm{Z}}$ is significantly enhanced. ${ }^{24}$ In practice, an incident angle of $21^{\circ}$ is used in LiPFIR experiments, which is slightly larger than the critical angle, and large enough to ensure total internal reflection for all mid-IR frequencies. As Figure $1 \mathrm{~b}$ indicates, the 
metallic AFM tip further amplifies the evanescent field, and the laser-induced photothermal expansion will occur at a nanometre-size hotspot underneath the tip, providing a high spatial resolution.

Figure 1e shows an experimentally collected cantilever deflection curve that contains photothermal expansion signals caused by IR absorptions. The repetition rate of the pulse train is adjusted to maximize the oscillation amplitude of the cantilever from consecutive photothermal expansions, possibly due to the enhancement from matching the mechanical resonance of the cantilever. Figure 1f shows the zoomed-in region of the laser-induced oscillations. A polynomial fit ( $4^{\text {th }}$ order) is performed to remove the slow varying curvature of the cantilever deflection in Figure $1 \mathrm{f}$ to retrieve pure oscillations at the repetition frequency of the laser pulse (Figure 1g). This procedure bypasses the self-reference scheme of the original PFIR microscopy ${ }^{13,}{ }^{37}$ which doubles the signal acquisition speed. The amplitude of the cantilever oscillations is extracted by fast Fourier transform (FFT). The resulting peak corresponding to the laser repetition rate is integrated and used as the LiPFIR signal (Figure 1h). IR nano-imaging is achieved by scanning the AFM tip over the sample surface while registering the LiPFIR signal with a fixed laser frequency; nanospectroscopy is obtained by parking the AFM tip at a desirable location on the sample and sweeping the laser frequency from the QCL.

\section{Identification of Nanoscale Domains of Polymer Blends}

Polymer blends formed by spontaneous phase separation serve as a model of soft matter to demonstrate the imaging and spectroscopy capability of LiPFIR microscopy. Figure 2a shows the topography of the polymer blend film (250-nm thick) formed by polystyrene (PS) and polymethyl methacrylate (PMMA) measured in heavy water. Two types of domains are 
noticeable in the topography: higher circular islands and a lower matrix region. Subjected to preparation conditions, spin-coated PS:PMMA blends can take different phase-separated geometries that cannot simply be distinguished from the topography. IR nano-imaging is required to perform chemical identification. To do so, the incident laser of the LiFPIR microscopy is tuned to IR frequencies that correspond to vibrational resonances of PS and PMMA, respectively. The resulting IR images are displayed in Figure 2b-c. At $1492 \mathrm{~cm}^{-1}$, PS domains exhibit a larger photothermal expansion signal as aromatic $\mathrm{C}=\mathrm{C}$ bonds absorb strongly at this frequency. At $1725 \mathrm{~cm}^{-1}$, PMMA domains are highlighted due to the absorption of the carbonyl $\mathrm{C}=\mathrm{O}$ vibrations. Figures 2b-c allow chemical identification of the domain compositions of polymer submerged in the liquid phase: the higher islands in the PS:PMMA blend polymer film are PMMA domains, and the lower matrix is the PS domain.

Point spectra collected with LiPFIR are shown in Figure 2d. When the AFM tip is fixed on circular islands, the averaged spectrum shows IR signatures of PMMA. Besides a strong carbonyl absorption peak at $1725 \mathrm{~cm}^{-1}$, there are also two peaks at $1380 \mathrm{~cm}^{-1}(\alpha$-methyl group vibration) and $1445 \mathrm{~cm}^{-1}$ (methyl $\mathrm{C}-\mathrm{H}$ vibrations). In comparison, the averaged spectrum obtained from the PS domain shows absorption peaks at $1450 \mathrm{~cm}^{-1}, 1495 \mathrm{~cm}^{-1}$, and $1601 \mathrm{~cm}^{-1}$, all of which are from aromatic $\mathrm{C}=\mathrm{C}$ vibrations of PS molecules. The distinctive spectra for PS and PMMA nanodomains indicate the unambiguous chemical sensitivity of LiPFIR. Note that the carbonyl peak at $1725 \mathrm{~cm}^{-1}$ of PMMA is also present in the PS domain, which is likely caused by bulk absorption of PMMA. The same effect was also observed in PS-b-PMMA copolymers with standard air phase PFIR, ${ }^{13}$ as well as in PiFM in the air. ${ }^{12}$ These results demonstrate that LiPFIR is capable of distinguishing multi-component polymer domains through both IR imaging and spectroscopy. The signal-to-noise ratio (relative peak height divided by the standard deviation of 
the background) of the $1725 \mathrm{~cm}^{-1}$ peak of the untreated PMMA spectrum is calculated to be 74 (see Supplementary Fig. S1), which is comparable to the signal to noise ratio of 70 obtained on PMMA using PTIR in water without plasmonic enhancement. ${ }^{25}$ As a demonstration of dynamic imaging in tracking the change of the absorption map in real time, we have also performed LiPFIR imaging in Supplementary Fig. S2 on monitoring surface reorganizations of PS:PMMA polymer domains induced by ethanol as the function of time, which exhibits the early stage of polymer swelling and delamination.

\section{Investigation of Reaction Sites of Click Chemistry}

Click chemistry offers a reliable way to synthesize chemicals with functional groups through the cycloaddition between azides and alkynes in water. ${ }^{38}$ In click chemistry, one of the most common strategies is copper-catalyzed azide-alkyne cycloaddition (CuAAC), where copper(I) is utilized as the catalyst. ${ }^{39}$ CuAAC has been utilized widely in organic synthesis, ${ }^{39}$ polymer chemistry, ${ }^{40}$ medical applications, ${ }^{41}$ and bioconjugation labeling. ${ }^{42}$ Spatially detecting the location of the CuAAC process at the nanoscale provides indications to the site-specific reactivity, which is important in heterogeneous materials and complex biological systems. Here, we utilize the LiPFIR microscopy to pinpoint reaction sites of the heterogeneous (solid/liquid) CuAAC in water.

Figure 3a illustrates the reaction process of the CuAAC between the azide-terminated PMMA (PMMA-N3) and the alkyne-functionalized biotin through polyethylene glycol chains (biotin-PEG-alkyne). The PMMA-N3 has a very similar absorptive profile to the regular PMMA in the range of $900-1800 \mathrm{~cm}^{-1}$, and FTIR spectra of PMMA-N3 and regular PMMA are shown in Supplementary Fig. S3. Similar to the PS:PMMA blend mixture in Figure 2, PS:PMMA-N3 
blend mixture is spin-coated on the prism surface to form a solid polymer film, and its topography is shown in Supplementary Fig. S4a. Then, $40 \mu \mathrm{L}$ of the reaction solution containing biotin-PEG-alkyne, $\mathrm{CuSO}_{4}$ and $\mathrm{C}_{6} \mathrm{H}_{7} \mathrm{NaO}_{6}$ is dropped on the polymer to start the CuAAC reaction. To examine the change of polymer morphology induced by the CuAAC, the prism surface is rinsed thoroughly with water after $90 \mathrm{~min}$ and air-dried. The polymer surface is then examined by the regular PFIR in the air in Supplementary Fig. S5. Several protrusions with lateral sizes less than $1 \mu \mathrm{m}$ on the PMMA-N3 domains are found in the topography, which are proved to be CuAAC reaction sites through IR imaging and spectroscopy. The PFIR spectrum collected from one reaction site (indicated in Supplementary Fig. S4c-d) shown in Figure $3 g$ exhibits a strong absorption peak of PEG at $1100 \mathrm{~cm}^{-1}$, confirming the existence of biotin-PEGalkyne at reaction sites. A full spectrum comparison performed in the air phase is provided in Supplementary Fig. S5e, where the amide I absorption of biotin is also observed.

Then, LiPFIR is used to locate the CuAAC reaction site in water in situ. After starting the reaction, the same sample area is scanned by LiPFIR intermittently without drying and LiPFIR images at $1100 \mathrm{~cm}^{-1}$ are captured. After 24 hours, a small protrusion area ( $200 \mathrm{~nm}$ in diameter) is located on the PMMA-N3 domain in Figure 3b. In Figure 3c, the adhesion map shows this protrusion area has lower adhesion compared with that of adjacent PMMA-N3 domains. Figure $3 \mathrm{~d}$ indicates four small bright spots at $1100 \mathrm{~cm}^{-1}$ around the protrusion area, while under the PMMA-N3 absorbing frequency of $1160 \mathrm{~cm}^{-1}$ in Figure 3e, these spots remain dark. Two point spectra displayed in Figure $3 \mathrm{f}$ are collected from one of four spots and unreacted PMMA-N3 surface nearby. A clear signature of PEG absorption at $1100 \mathrm{~cm}^{-1}$ is observed for the reaction site, while for the unreacted PMMA-N3, only the peak at $1160 \mathrm{~cm}^{-1}$ is present. The signal to noise ratio of the $1160 \mathrm{~cm}^{-1}$ peak for the PMMA-N3 domain in water is estimated to be 37 (see 
Supplementary Fig. S6), which is smaller than that of 191 obtained on the PMMA-N3 domain in the air phase. From a cross-sectioning profile of one hotspot in Figure 3d, the detection limit of LiPFIR is estimated to be $1.2 \times 10^{3}$ biotin-PEG-alkyne molecules (Supplementary Fig. S7). While the air-phase PFIR provides the IR spectrum of the reaction product with excellent quality (Figure 3g), LiPFIR is capable of locating and distinguishing the CuAAC reaction site in operando, without drying the sample or interrupting the reaction. The joint usage of LiPFIR with CuAAC reaction will facilitate the biological investigations that use tags with click chemistry to pinpoint objects of interest at solid/liquid interface.

\section{Quantification of Spatial Resolution of LiPFIR}

Bovine serum albumin (BSA) is widely used as a model protein in many biological processes and applications. ${ }^{43,44}$ PTIR has been utilized in revealing the secondary structures of proteins. ${ }^{25,45}$ Here, we use LiPFIR to perform nano-imaging of the BSA sample in water. At room temperature, BSA proteins tend to form fibril structures in solution. ${ }^{46,47}$ To immobilize the BSA, the germanium surface is silanized with APTES (see Methods and Materials). AFM topography of a segment of a BSA fibril in $0.1 \times$ PBS buffer is shown in Figure 4a. This single BSA fibril is $150 \mathrm{~nm}$ in height and $350 \mathrm{~nm}$ in width. LiPFIR images at $1630 \mathrm{~cm}^{-1}$ (amide I band) are shown in Figure 4b-d in water and heavy water environments. In water, a large IR signal along the BSA fibril is observed, which distinguishes the protein fibril from the substrate. The spotty signals on the substrate are observed at small crevices formed on the rough germanium surface, where hot spots are created by strong field enhancements, leading to additional signal background from the photothermal expansion of the fluid. In heavy water, amide I band IR absorption is still present in the fibril. Another observation from Figure $4 c$ is that a small region at the left terminal (indicated by an arrow) of the BSA fibril exhibits much reduced IR signal at $1630 \mathrm{~cm}^{-1}$. Such a 
difference in IR responses indicates a heterogeneous change of the protein secondary structure at the left terminal of the fibril when put in heavy water.

To estimate the spatial resolution of LiPFIR imaging, sectioned signal profiles from different locations of the edge of BSA fibril are plotted in Figure 3d, and spatial resolutions of 8 - $12 \mathrm{~nm}$ are obtained for LiPFIR in water. By comparison, contact mode AFM-IR in water recently achieved a $20 \sim 25 \mathrm{~nm}$ resolution, ${ }^{24,}{ }^{25}$ from a $100-\mathrm{nm}$ spatial resolution in its early implementation. ${ }^{14}$

LiPFIR preserves the surface integrity of the sample during the measurement and allows for repeated measurements of the same region while changing the composition of the fluid. The topography of the BSA fibril stays the same after 8 hours of scans (Supplementary Fig. S8). The H-D exchange is revealed by spectroscopic results obtained in situ on the same location of BSA fibril in water and heavy water. Measured IR spectra are displayed in Supplementary Fig. S9. When in heavy water, since the majority of amide II band absorption is from N-H bonds in proteins, the replacement of N-H with N-D bonds redshifts the amide II absorption from 1550 to $1460 \mathrm{~cm}^{-1} \cdot{ }^{48}$ As a result, a strong amide II band of the BSA fibril is observed in heavy water, which centers around $1460 \mathrm{~cm}^{-1}$ and indicates the completion of H-D exchange.

\section{Investigating Yeast Budding Site in the Liquid Phase}

Avoiding the destructive shear force means that the LiPFIR microscopy is suitable for imaging highly uneven soft matter samples with large height variations in water, such as biological cells. To demonstrate the capability of LiPFIR in imaging large and soft biological samples, we use it to examine the chemical composition of large cell wall particles derived from the yeast Saccharomyces cerevisiae. These cell wall particles are often referred to as zymosan 
particles, which represent a group of naturally occurring immunomodulators for therapeutic applications. ${ }^{49-51}$

Zymosan particles exhibit different shapes in the air and the aqueous phase. Figure 5a shows the topography of a dried zymosan particle in the air. The dried zymosan particle exhibits a flattened disk-like shape with an average height of $\sim 250 \mathrm{~nm}$. In the dried form, the nucleus of the original yeast becomes noticeable and appears protruded from the center of the zymosan particle. ${ }^{52,53}$ Another noticeable feature on the zymosan particle is the presence of a bud scar on the lower right. The bud scar is a ring-shaped fracture formed during the budding (division) process of yeast cells. We first perform air-phase peak force IR microscopy on this zymosan particle. The sample is illuminated at $1030 \mathrm{~cm}^{-1}$ (C-O-C vibrations) and $1630 \mathrm{~cm}^{-1}$ (amide I band), resolving the spatial distribution of polysaccharides and proteins spatially. ${ }^{54}$ As shown in Figure 5b-c, the IR image at $1030 \mathrm{~cm}^{-1}$ suggests that polysaccharide components, which mainly included $\beta$-glucan and mannan, are evenly distributed across the zymosan surface. In comparison, the IR image at $1630 \mathrm{~cm}^{-1}$ clearly shows the uneven distribution of proteins. Proteins are enriched in multiple scattered spots, the nucleus, and the bud scar. Other locations of the zymosan particle also exhibit a small IR signal at $1630 \mathrm{~cm}^{-1}$, indicating proteins are present together with polysaccharides on the rest area of the zymosan surface.

Dried zymosan particles, after immersed in heavy water, restore to their native ellipsoidal shape. Figure 5d shows the topography of two zymosan particles in heavy water with heights of more than $1 \mu \mathrm{m}$. The height of zymosan particles increases from 200 300 $\mathrm{nm}$ in the air to up to $\sim 1.8 \mu \mathrm{m}$ in heavy water, and the large nucleus observed in the air disappears because it is now enclosed inside the shell of the engorged zymosan particle. An unusual aspect of the AFM image of Figure $5 d$ is that it captures two zymosan particles in the 
budding process with a septum formed in between. LiPFIR images are displayed in Figure 5e-f. In Figure 5e, an even distribution of polysaccharides is observed. In Figure 5f, the distribution of proteins also appears uniform, and slightly stronger signals present at peripherals because the evanescent IR field is stronger at lower heights from the Ge/water interface. Interestingly, strong $1630 \mathrm{~cm}^{-1}$ IR absorptions that correspond to amide I are observed on a cluster of material close to the budding site, as indicated by the arrow in Figure 5f. The cluster is connected with the budding septum from the bottom, as revealed by the LiPFIR images. The measurement suggests that the septum in the budding process contains molecules of amide I band, e.g., protein or chitin..$^{55}$

To further confirm the chemical composition of the cluster in the vicinity of the budding site, four point spectra are collected and shown in Figure 5g. Positions 1 and 3 are taken on the two zymosan particles, and position 2 is on the septum. Positions 1-3 show similar IR signatures of polysaccharides: The peak at $1190 \mathrm{~cm}^{-1}$ is attributed to the absorption of heavy water inside the zymosan particles; small IR absorptions between $1000 \sim 1100 \mathrm{~cm}^{-1}$ and the peak at $1220 \mathrm{~cm}^{-1}$ are due to the $\mathrm{C}-\mathrm{O}$ stretching, and the peak at $1430 \mathrm{~cm}^{-1}$ is attributed to $\mathrm{C}-\mathrm{H}$ bending. The spectrum collected on position 4, which is the bright spot observed at $1630 \mathrm{~cm}^{-1}$, however, does not show discernable signatures of polysaccharide and chitin, which absorb around 1000 1100 $\mathrm{cm}^{-1}$. Instead, a sole amide I band around $1630 \mathrm{~cm}^{-1}$ is observed. This finding confirms that the highlighted cluster observed at $1630 \mathrm{~cm}^{-1}$ is mainly composed of proteins, and a medium band around $1430 \mathrm{~cm}^{-1}$ is attributed to the H-D exchanged amide II band. The proteins in the cluster may participate in the synthesis of chitins during the budding process, as suggested by the literature. $^{56}$

\section{Discussion}


The penetration depth of the evanescent field gives an upper boundary of the height of the sample that can be measured by the LiPFIR microscopy. The zymosan particles of Figure 5 are a high sample with a height of $1.8 \mu \mathrm{m}$. The penetration depth $d$ of evanescent field established by the total internal reflection at the germanium/water interface can be calculated using the following equation:

$$
d=\frac{\lambda}{4 \pi \sqrt{n_{1}^{2} \sin ^{2} \theta-n_{2}^{2}}}
$$

where $n_{1}$ and $n_{2}$ are refractive indices of germanium and water, respectively, $\lambda$ is light wavelength, and $\theta \quad$ is the incident angle. With estimated values of $n_{1}=4, n_{2}=1.36$, $\lambda=6.25 \mu \mathrm{m}$, and $\theta=20^{\circ}$, Equation 1 gives a penetration depth of $3.3 \mu \mathrm{m}$, which is long enough to induce the tip-enhanced light field at the top of the zymosan particles.

The ability of LiPFIR represents an advancement for the nanoscale IR microscopy. The non-destructiveness and label-free imaging capability of LiPFIR enable the in situ measurement of the physical transformations and chemical reactions at the solid/liquid interface. Wide varieties of industrially significant chemical reactions happen in the liquid phase and solid/liquid interfaces, where the mass transportation capabilities are more favorable than those in the air phase. Most biological processes at the molecular level take place in aqueous conditions, too, where the biomolecules are in native states. The LiPFIR microscopy can extend the standard PFIR into these two crucial areas of research.

The non-destructiveness of LiPFIR stems from the precise control of the maximal tipsample forces in the PFT feedback. In the liquid phase, intermolecular forces between the solvent molecule and sample molecule destabilize the sample surface, making the surface more 
vulnerable to mechanical scratches by the AFM tip than in the air phase. To preserve the integrity of the sample surface, the lateral shear force between the AFM tip and sample should be minimized. In the PFT mode, the AFM tip only momentarily contacts with the sample surface for tens of $\mu$ s in every PFT cycle. The shear force only builds up momentarily during the contact and is released when the tip-sample detaches in each PFT cycle. No large lateral shear force is present in LiPFIR. A direct comparison between the contact mode (where the lateral shear force is present) and peak force tapping mode in water is shown in Supplementary Fig. S10. Consecutive measurements with LiPFIR over an extended period ( $>8$ hours) on the protein fibrils do not induce any noticeable changes in the topography or disruptions of the sample surface (Supplementary Figure S8). In the zymosan studies of Figure 5, the zymosan particle filled with heavy water exhibit a height of $1.8 \mu \mathrm{m}$ over a lateral dimension of $5 \mu \mathrm{m}$. The zymosan particle is also very soft, with a measured reduced Young's modulus of only $0.1 \mathrm{MPa}$ in heavy water (Supplementary Figure S11). As an analogy, the zymosan in the liquid phase behaves like a water-filled balloon, and the nanoscopic AFM tip acts as a needle. Scratching the needle along the water-filled balloon would easily break it. Therefore, this is a type of sample that would be challenging for the contact mode AFM. The cantilever deflection setpoint should be very small to reduce the lateral force; the gain of the AFM feedback loop has to be large to cope with large height variations. However, a large feedback gain based on a low set point would amplify noise in the AFM scan, leading to possible sample damages when the AFM tip is out of topography feedback. In the LiFPIR, we use a high tapping amplitude of $100 \mathrm{~nm}$ and a low peak force setpoint of $1 \mathrm{nN}$ to measure the zymosan sample, which provided stable topography feedback and adequate IR imaging. 
The LiPFIR microscopy provides a spatial resolution of $\sim 10 \mathrm{~nm}$, which is slightly worse than the PFIR microscopy in the air phase. ${ }^{54,57}$ In comparison, the state-of-the-art resonantly enhanced contact mode AFM-IR techniques (i.e., REINS and resonantly enhanced PTIR) can achieve $20 \sim 25 \mathrm{~nm}$ spatial resolution in the liquid. ${ }^{24,} 25$ LiPFIR gives a 2.5-time improved spatial resolution for liquid-phase IR microscopy, albeit at the expense of a lower signal strength than REINS or resonantly enhanced PTIR due to the fact that peak force tapping mode provides intermittently tip-sample contact, instead of constant contact in the contact mode. The improvement of spatial resolution of LiPFIR is due to the small area of contact in the peak force tapping when the peak force setpoint is kept low. The difference of mechanical properties across the composition boundary also improves the spatial contrast in the photothermal detection of IR absorption.

Recent development of total internal reflection scattering-type scanning near-field optical microscopy (s-SNOM) in the liquid achieves a spatial resolution of 30-80 nm. ${ }^{26,27}$ However, in order for s-SNOM to conveniently deliver IR imaging, a narrowband light source has to be used. $^{27}$ Furthermore, performing spectroscopy with narrowband light sources in s-SNOM for interferometric detection is time-consuming. To efficiently obtain IR imaging and spectroscopy for s-SNOM, both broadband and narrowband IR sources are often required. ${ }^{29}$ In comparison, LiPFIR and other photothermal AFM-IR techniques, in general, can readily acquire both IR images and point spectra with one set of frequency tunable narrowband laser source, avoiding complex interferometric detection apparatus that requires a high level of operational precision on the optical alignment.

\section{Conclusion}


The compatibility with liquid-phase environments, the high spatial resolution, and the chemical sensitivity enables the LiPFIR microscopy to be a versatile platform for monitoring transformations at solid/liquid interfaces non-intrusively in a label-free manner. The LiPFIR microscope with the fluid perfusion chamber permits the nano-IR imaging and spectroscopy on spatial locations of interest under different liquid compositions that cause physical transformation and/or chemical reactions. This capability allows for in situ and in operando investigations over a broad range of solid/liquid interfacial chemical and biological processes, such as heterogeneous chemical reactions and catalysis, ${ }^{58}$ polymer membranes for biofouling mitigation, ${ }^{59}$ drug-protein interactions, ${ }^{60}$ as well as chemical sensing through mid-IR polaritons. ${ }^{22}$

\section{Methods and Materials}

\section{LiPFIR apparatus}

A waveform of PFT frequency was routed out from an AFM controller (Nanoscope V, Bruker) to synchronize laser pulses with peak force tapping (PFT). This waveform was then amplified and connected to a lock-in amplifier (MFLI, Zurich Instruments) working as a phase lock loop, which generates a TTL waveform at the same PFT frequency. The TTL waveform was used to trigger a function generator (HDG2022B, Hantek) working in the burst mode, which generated a train of electric pulses that were used to trigger the QCL. The repetition rate of the pulse train was modified by changing the parameters of the function generator, and the duration (which is proportional to the energy) of each laser pulse was controlled by the QCL program. The laser pulse duration used in this study ranged from 100-1000 ns (the energy of each pulse corresponds to $~ 20-200 \mathrm{~nJ}$ ), depending on the signal strength and quality. The cantilever deflection signal is acquired by a digitizer (PXI-5122, National Instruments) and processed through a program 
written in LabVIEW (National Instruments) in real-time. Analog outputs from the LabVIEW program were routed into the AFM controller to form IR images together with the mechanical properties from the force curve analysis of peak force tapping mode (PeakForce QNM, Bruker). In the spectroscopy mode, the scan size is set to zero so that the tip is parked on the spot of measurement. The deflection waveform was recorded along with the laser frequency. The data was then processed by MATLAB to produce an IR spectrum.

The laser repetition rate for LiPFIR measurements shown in the manuscript was adjusted case by case, as the contact resonance of the cantilever is affected by many factors, including the type of AFM cantilever, the sample itself, and tapping conditions. In addition, since multiple contact resonances of the cantilever are present in the aqueous phase, one needs to choose the appropriate contact resonance that provides the best signal quality. For most LiPFIR experiments, Au-coated AFM tips (HQ:NSC19/Cr-Au, MikroMasch) with an end radius of around $25 \mathrm{~nm}$ and 10-20 laser pulses were used. For the measurement of polymer blends in heavy water, the pulse repetition rate of the pulse train was tuned to $123 \mathrm{kHz}$. The LiPFIR signal was obtained from the FFT peak at $123 \mathrm{kHz}$, as well. For the measurement of polymer blends in ethanol, the pulse repetition rate was set as $264 \mathrm{kHz}$. The LiPFIR signal was obtained from the FFT peak at $86 \mathrm{kHz}$. For the H-D exchange of the BSA fibril, the pulse repetition rate and the FFT peak were both at $262 \mathrm{kHz}$. For measuring zymosan particles, a new Pt-coated tip (PFTUNA, Bruker) with the end radius of $25 \mathrm{~nm}$ was used, and the laser repetition rate and FFT peak were both at $210 \mathrm{kHz}$. For the measurement of the click-reaction, Au-coated tips were used. The laser repetition rate and the LiPFIR signal were both at $220 \mathrm{kHz}$. The duration of each laser pulse was adjusted between 100-1000 ns to optimize the imaging quality. 
For all measured samples, the PFT frequency was set as $1 \mathrm{kHz}$, the peak force setpoint was set in a range of 3-15 nN, and the PFT amplitude was 100-150 nm.

\section{Simulation and Calculation}

The simulation of the tip-enhanced evanescent field in Figure 1b was done by the program Lumerical FDTD (Lumerical Inc.). In the simulation, a gold-coated tip with an end radius of $30 \mathrm{~nm}$ was placed $3 \mathrm{~nm}$ above the germanium surface. A P-polarized (polarized inplane, as shown in Figure 1a-b) plane wave was used to simulate the laser that generated the total internal reflection with an incident angle of $20^{\circ}$. The critical angle was calculated using Snell's law, and the field strength $E_{z}$ was calculated using the formula in the literature. ${ }^{36}$

\section{Preparations of polymer blends and click chemistry}

Polystyrene (PS, Mw: 192000, Sigma-Aldrich) and polymethyl methacrylate (PMMA, Mw: 120000, Sigma-Aldrich) were dissolved in toluene to form a $12 \mathrm{mg} / \mathrm{mL}$ solution (the weight ratio of PS:PMMA is 1:1.5). Then, the blended polymer sample was made by spin-coating $50 \mu \mathrm{L}$ of the solution onto the 3- $\mathrm{cm}^{2}$ top surface of a germanium prism at $2500 \mathrm{rpm}$ for $1 \mathrm{~min}$ by a spincoat machine (KW-4A, MicroNano Tools) conjoined with a customized adaptor for the prism.

For measurements of click reactions, the germanium prism was spin-coated with a mixture in toluene of $\omega$-azide-terminated PMMA (P41623-MA-N3, Polymer Source, MW: 1000) and polystyrene (Sigma Aldrich, MW: 192000) with the concentration of $17 \mathrm{mg} / \mathrm{mL}$ and 25 $\mathrm{mg} / \mathrm{mL}$, respectively. The reaction solution is made of $\mathrm{CuSO}_{4}(1 \mathrm{mM}$, prepared from copper(II) sulfate pentahydrate, Sigma Aldrich), $\mathrm{C}_{6} \mathrm{H}_{7} \mathrm{NaO}_{6}(7 \mathrm{mM}$, freshly prepared from (+)-sodium Lascorbate, Sigma Aldrich) and biotin-PEG4-alkyne (7 mM, Sigma Aldrich). After locating a 
scanning position with AFM in the air, the cantilever was first retracted from the surface by 40 $\mu \mathrm{m}$, and the fluid chamber is filled with a total of $40 \mu \mathrm{L}$ reaction solution by a syringe.

\section{Preparation of BSA fibrils}

Bovine serum albumin (99\%, Sigma-Aldrich) was dissolved in $1 \times$ PBS in a concentration of $10 \mathrm{mg} / \mathrm{mL}$ and stored in a $4^{\circ} \mathrm{C}$ fridge. The BSA solution was then diluted with deionized water to $1 \mathrm{mg} / \mathrm{mL}$. The BSA solution was kept at room temperature for a month to form BSA fibrils, and then $40 \mu \mathrm{L}$ of it was drop-casted on the Ge surface. The Germanium prism surface was functionalized with (3-aminopropyl)triethoxysilane (APTES) (Sigma-Aldrich) to immobilize proteins. The prism surface was first washed by soaking it in chloroform three times and 5 minutes for each time. Then, the prism was incubated with $45 \mu \mathrm{L}$ of APTES and $15 \mu \mathrm{L}$ of triethylamine (Sigma-Aldrich) in a $5 \mathrm{~L}$ desiccator filled with argon gas for 2 hours. After two hours, the vials containing APTES and triethylamine were removed, and the desiccator was sealed for another 8-hour incubation.

\section{Preparation of zymosan particles}

Zymosan particles were purchased from InvivoGen (San Diego, CA). To prepare samples

for LiPFIR measurements, $20 \mu \mathrm{L}$ of the aqueous solution containing zymosan particles at 20 $\mu \mathrm{g} / \mathrm{mL}$ concentration was dropped and dried on the Germanium prism. In LiPFIR measurement, the sample was immersed in heavy water without using immobilization procedure.

\section{Acknowledgments}

H.W. is thankful for Yuyuan Zhou for the help of the silanization of the germanium prism and Wenpeng Cao for the help of providing BSA proteins. X. G. X. would like to thank the support 
from Beckman Young Investigator Award from the Arnold and Mabel Beckman Foundation and the Sloan Research Fellowship from the Alfred P. Sloan Foundation. H. W. and X. G. X. would also like to thank the support from the National Science Foundation, award number CHE 1847765. W. L. and Y. Y. acknowledge support from the National Institute of General Medical Sciences of NIH under Award Number R35GM124918. The content is solely the responsibility of the authors and does not necessarily represent the official views of NIH.

\section{Author contribution}

X. G. X. designed the idea of LiPFIR. H.W. and X.G.X built the experimental apparatus. H.W. performed the numerical simulation, collected the data, and performed data analysis. Q. X. prepared the sample for the click reaction measurement. H.W., J. M. G., and X. G. X. wrote the manuscript. W. L. and Y. Y. provided the zymosan sample. X. G. X guided the overall research.

\section{References}

1. Abbe, E., Beiträge zur Theorie des Mikroskops und der mikroskopischen Wahrnehmung. Archiv für mikroskopische Anatomie 1873, 9 (1), 413-418.

2. Zhang, D.; Li, C.; Zhang, C.; Slipchenko, M. N.; Eakins, G.; Cheng, J.-X., Depth-resolved mid-infrared photothermal imaging of living cells and organisms with submicrometer spatial resolution. Science Advances 2016, 2 (9), e1600521.

3. Shi, J.; Wong, T. T. W.; He, Y.; Li, L.; Zhang, R.; Yung, C. S.; Hwang, J.; Maslov, K.; Wang, L. V., High-resolution, high-contrast mid-infrared imaging of fresh biological samples with ultravioletlocalized photoacoustic microscopy. Nature Photonics 2019, 13 (9), 609-615.

4. Centrone, A., Infrared Imaging and Spectroscopy Beyond the Diffraction Limit. Annual Review of Analytical Chemistry 2015, 8 (1), 101-126.

5. Richards, D.; Zayats, A.; Keilmann, F.; Hillenbrand, R., Near-field microscopy by elastic light scattering from a tip. Philosophical Transactions of the Royal Society of London. Series A: Mathematical, Physical and Engineering Sciences 2004, 362 (1817), 787-805.

6. Zrimsek, A. B.; Chiang, N.; Mattei, M.; Zaleski, S.; McAnally, M. O.; Chapman, C. T.; Henry, A.-I.; Schatz, G. C.; Van Duyne, R. P., Single-Molecule Chemistry with Surface- and Tip-Enhanced Raman Spectroscopy. Chemical Reviews 2017, 117 (11), 7583-7613.

7. Wang, X.; Huang, S.-C.; Huang, T.-X.; Su, H.-S.; Zhong, J.-H.; Zeng, Z.-C.; Li, M.-H.; Ren, B. J. C. S. R., Tip-enhanced Raman spectroscopy for surfaces and interfaces. 2017, 46 (13), 4020-4041.

8. Mahapatra, S.; Li, L.; Schultz, J. F.; Jiang, N., Tip-enhanced Raman spectroscopy: Chemical analysis with nanoscale to angstrom scale resolution. The Journal of Chemical Physics 2020, 153 (1), 010902. 
9. $\quad$ Lipiec, E.; Kaderli, J.; Kobierski, J.; Riek, R.; Skirlińska-Nosek, K.; Sofińska, K.; Szymoński, M.; Zenobi, R., Nanoscale hyperspectral imaging of amyloid secondary structures in liquid. Angewandte Chemie International Edition 2020, n/a (n/a).

10. Knoll, B.; Keilmann, F., Enhanced dielectric contrast in scattering-type scanning near-field optical microscopy. Optics Communications 2000, 182 (4), 321-328.

11. Dazzi, A.; Prater, C. B., AFM-IR: Technology and Applications in Nanoscale Infrared Spectroscopy and Chemical Imaging. Chemical Reviews 2017, 117 (7), 5146-5173.

12. Nowak, D.; Morrison, W.; Wickramasinghe, H. K.; Jahng, J.; Potma, E.; Wan, L.; Ruiz, R.; Albrecht, T. R.; Schmidt, K.; Frommer, J., Nanoscale chemical imaging by photoinduced force microscopy. Science Advances 2016, 2 (3), e1501571.

13. Wang, L.; Wang, H.; Wagner, M.; Yan, Y.; Jakob, D. S.; Xu, X. G., Nanoscale simultaneous chemical and mechanical imaging via peak force infrared microscopy. Sci. Adv. 2017, 3 (6), e1700255.

14. Mayet, C.; Dazzi, A.; Prazeres, R.; Allot, F.; Glotin, F.; Ortega, J. M., Sub-100 nm IR spectromicroscopy of living cells. Optics Letters 2008, 33 (14), 1611-1613.

15. Dazzi, A.; Prazeres, R.; Glotin, F.; Ortega, J. M., Local infrared microspectroscopy with subwavelength spatial resolution with an atomic force microscope tip used as a photothermal sensor. Optics Letters 2005, 30 (18), 2388-2390.

16. Lu, F.; Jin, M.; Belkin, M. A., Tip-enhanced infrared nanospectroscopy via molecular expansion force detection. Nature Photonics 2014, 8 (4), 307-312.

17. Almajhadi, M.; Wickramasinghe, H. K., Contrast and imaging performance in photo induced force microscopy. Optics Express 2017, 25 (22), 26923-26938.

18. Almajhadi, M. A.; Uddin, S. M. A.; Wickramasinghe, H. K., Observation of nanoscale optomechanical molecular damping as the origin of spectroscopic contrast in photo induced force microscopy. Nature Communications 2020, 11 (1), 5691.

19. Jahng, J.; Potma, E. O.; Lee, E. S., Tip-Enhanced Thermal Expansion Force for Nanoscale Chemical Imaging and Spectroscopy in Photoinduced Force Microscopy. Analytical Chemistry 2018, 90 (18), 11054-11061.

20. Jahng, J.; Potma, E. O.; Lee, E. S., Nanoscale spectroscopic origins of photoinduced tip-sample force in the midinfrared. Proceedings of the National Academy of Sciences 2019, 116 (52), 26359-26366.

21. Rosa-Zeiser, A.; Weilandt, E.; Hild, S.; Marti, O., The simultaneous measurement of elastic, electrostatic and adhesive properties by scanning force microscopy: pulsed-force mode operation. Measurement Science and Technology 1997, 8 (11), 1333.

22. Jakob, D. S.; Wang, H.; Zeng, G.; Otzen, D. E.; Yan, Y.; Xu, X. G., Peak Force Infrared-Kelvin Probe Force Microscopy. Angewandte Chemie International Edition 2020, 59 (37), 16083-16090.

23. Vongsvivut, J.; Pérez-Guaita, D.; Wood, B. R.; Heraud, P.; Khambatta, K.; Hartnell, D.; Hackett, M. J.; Tobin, M. J., Synchrotron macro ATR-FTIR microspectroscopy for high-resolution chemical mapping of single cells. Analyst 2019, 144 (10), 3226-3238.

24. Jin, M.; Lu, F.; Belkin, M. A., High-sensitivity infrared vibrational nanospectroscopy in water. Light: Science \& Applications 2017, 6 (7), lsa201796.

25. Ramer, G.; Ruggeri, F. S.; Levin, A.; Knowles, T. P. J.; Centrone, A., Determination of polypeptide conformation with nanoscale resolution in water. ACS Nano 2018, 12 (7), 6612-6619.

26. O’Callahan, B. T.; Park, K.-D.; Novikova, I. V.; Jian, T.; Chen, C.-L.; Muller, E. A.; ElKhoury, P. Z.; Raschke, M. B.; Lea, A. S., In Liquid Infrared Scattering Scanning Near-Field Optical Microscopy for Chemical and Biological Nanoimaging. Nano Letters 2020, 20 (6), 4497-4504.

27. Pfitzner, E.; Heberle, J., Infrared Scattering-Type Scanning Near-Field Optical Microscopy of Biomembranes in Water. The Journal of Physical Chemistry Letters 2020, 11 (19), 8183-8188.

28. Wang, H.; Janzen, E.; Wang, L.; Edgar, J. H.; Xu, X. G., Probing Mid-Infrared Phonon Polaritons in the Aqueous Phase. Nano Letters 2020, 20 (5), 3986-3991.

29. Virmani, D.; Bylinkin, A.; Lopez, I. D.; Janzen, E.; Edgar, J. H.; Hillenbrand, R., Amplitudeand phase-resolved nano-imaging and nano-spectroscopy of polaritons in liquid environment. arXiv preprint arXiv:2010.06229 2020. 
30. Li, J.; Pang, J.; Yan, Z.-d.; Jahng, J.; Li, J.; Morrison, W.; Liang, J.; Zhang, Q.-Y.; Xia, X.-H., Antenna enhancing infrared photoinduced force imaging in aqueous environment with super-resolution and hypersensitivity. arXiv preprint 2020, arXiv:.11555.

31. Cuberes, M. T.; Assender, H. E.; Briggs, G. A. D.; Kolosov, O. V., Heterodyne force microscopy of PMMA/rubber nanocomposites: nanomapping of viscoelastic response at ultrasonic frequencies. Journal of Physics D: Applied Physics 2000, 33 (19), 2347.

32. Alsteens, D.; Dupres, V.; Yunus, S.; Latgé, J.-P.; Heinisch, J. r. J.; Dufrêne, Y. F., Highresolution imaging of chemical and biological sites on living cells using peak force tapping atomic force microscopy. Langmuir 2012, 28 (49), 16738-16744.

33. Rico, F.; Su, C.; Scheuring, S., Mechanical mapping of single membrane proteins at submolecular resolution. Nano Letters 2011, 11 (9), 3983-3986.

34. Pittenger, B.; Erina, N.; Su, C., Quantitative mechanical property mapping at the nanoscale with PeakForce QNM. Application Note Veeco Instruments Inc. 2010, 1-12.

35. Johnson, K. L.; Woodhouse, J., Stick-slip motion in the atomic force microscope. Tribology Letters 1998, 5 (2), 155-160.

36. Milosevic, M., On the nature of the evanescent wave. Applied Spectroscopy 2013, 67 (2), 126131.

37. Wang, L.; Wagner, M.; Wang, H.; Pau-Sanchez, S.; Li, J.; Edgar, J. H.; Xu, X. G., Revealing Phonon Polaritons in Hexagonal Boron Nitride by Multipulse Peak Force Infrared Microscopy. Advanced Optical Materials 2020, 8 (5), 1901084.

38. Kolb, H. C.; Finn, M. G.; Sharpless, K. B., Click Chemistry: Diverse Chemical Function from a Few Good Reactions. Angewandte Chemie International Edition 2001, 40 (11), 2004-2021.

39. Hein, J. E.; Fokin, V. V., Copper-catalyzed azide-alkyne cycloaddition (CuAAC) and beyond: new reactivity of copper(i) acetylides. Chemical Society Reviews 2010, 39 (4), 1302-1315.

40. Meldal, M., Polymer "Clicking” by CuAAC Reactions. Macromolecular Rapid Communications 2008, 29 (12-13), 1016-1051.

41. Geva-Zatorsky, N.; Alvarez, D.; Hudak, J. E.; Reading, N. C.; Erturk-Hasdemir, D.; Dasgupta, S.; von Andrian, U. H.; Kasper, D. L., In vivo imaging and tracking of host-microbiota interactions via metabolic labeling of gut anaerobic bacteria. Nature Medicine 2015, 21 (9), 1091-1100.

42. McKay, Craig S.; Finn, M. G., Click Chemistry in Complex Mixtures: Bioorthogonal Bioconjugation. Chemistry \& Biology 2014, 21 (9), 1075-1101.

43. Zhang, W.; Zhao, Q.; Yuan, J., Porous polyelectrolytes: the interplay of charge and pores for new functionalities. Angewandte Chemie International Edition 2018, 57 (23), 6754-6773.

44. Comby, S.; Gunnlaugsson, T., Luminescent lanthanide-functionalized gold nanoparticles: exploiting the interaction with bovine serum albumin for potential sensing applications. ACS Nano 2011, 5 (9), 7184-7197.

45. Ruggeri, F. S.; Mannini, B.; Schmid, R.; Vendruscolo, M.; Knowles, T. P. J., Single molecule secondary structure determination of proteins through infrared absorption nanospectroscopy. Nature Communications 2020, 11 (1), 2945.

46. de la Arada, I.; Seiler, C.; Mäntele, W., Amyloid fibril formation from human and bovine serum albumin followed by quasi-simultaneous Fourier-transform infrared (FT-IR) spectroscopy and static light scattering (SLS). European Biophysics Journal 2012, 41 (11), 931-938.

47. Pearce, F. G.; Mackintosh, S. H.; Gerrard, J. A., Formation of amyloid-like fibrils by ovalbumin and related proteins under conditions relevant to food processing. Journal of Agricultural and Food Chemistry 2007, 55 (2), 318-322.

48. Blout, E.; De Loze, C.; Asadourian, A., The deuterium exchange of water-soluble polypeptides and proteins as measured by infrared spectroscopy. Journal of the American Chemical Society 1961, 83 (8), 1895-1900.

49. $\quad$ Pan, Y.; Li, X.; Kang, T.; Meng, H.; Chen, Z.; Yang, L.; Wu, Y.; Wei, Y.; Gou, M., Efficient delivery of antigen to DCs using yeast-derived microparticles. Scientific Reports 2015, 5, 10687. 
50. $\quad$ De Smet, R.; Demoor, T.; Verschuere, S.; Dullaers, M.; Ostroff, G. R.; Leclercq, G.; Allais, L.; Pilette, C.; Dierendonck, M.; De Geest, B. G., $\beta$-Glucan microparticles are good candidates for mucosal antigen delivery in oral vaccination. Journal of Controlled Release 2013, 172 (3), 671-678.

51. Novak, M.; Vetvicka, V., $\beta$-glucans, history, and the present: immunomodulatory aspects and mechanisms of action. Journal of Immunotoxicology 2008, 5 (1), 47-57.

52. Lombard, Y.; Giaimis, J.; Makaya-Kumba, M.; Fonteneau, P.; Poindron, P., A new method for studying the binding and ingestion of zymosan particles by macrophages. Journal of Immunological Methods 1994, 174 (1-2), 155-165.

53. Jawhara, S.; Habib, K.; Maggiotto, F.; Pignede, G.; Vandekerckove, P.; Maes, E.; Dubuquoy, L.; Fontaine, T.; Guerardel, Y.; Poulain, D., Modulation of intestinal inflammation by yeasts and cell wall extracts: strain dependence and unexpected anti-inflammatory role of glucan fractions. PloS One 2012, 7 (7).

54. Li, W.; Wang, H.; Xu, X. G.; Yu, Y., Simultaneous Nanoscale Imaging of Chemical and Architectural Heterogeneity on Yeast Cell Wall Particles. Langmuir 2020, 36 (22), 6169-6177.

55. Fleet, G. H., Composition and structure of yeast cell walls. In Current Topics in Medical Mycology, Springer: 1985; pp 24-56.

56. Cabib, E.; Durán, A., Synthase III-dependent chitin is bound to different acceptors depending on location on the cell wall of budding yeast. Journal of Biological Chemistry 2005, 280 (10), 9170-9179.

57. Jakob, D. S.; Wang, L.; Wang, H.; Xu, X. G., Spectro-Mechanical Characterizations of Kerogen Heterogeneity and Mechanical Properties of Source Rocks at $6 \mathrm{~nm}$ Spatial Resolution. Analytical Chemistry 2019, 91 (14), 8883-8890.

58. Fechete, I.; Wang, Y.; Védrine, J. C., The past, present and future of heterogeneous catalysis. Catalysis Today 2012, 189 (1), 2-27.

59. Gunari, N.; Brewer, L. H.; Bennett, S. M.; Sokolova, A.; Kraut, N. D.; Finlay, J. A.; Meyer, A. E.; Walker, G. C.; Wendt, D. E.; Callow, M. E.; Callow, J. A.; Bright, F. V.; Detty, M. R., The control of marine biofouling on xerogel surfaces with nanometer-scale topography. Biofouling 2011, 27 (2), 137149.

60. Ye, C.; Chi, H., A review of recent progress in drug and protein encapsulation: Approaches, applications and challenges. Materials Science and Engineering: C 2018, 83, 233-246. 
Figures

(a)

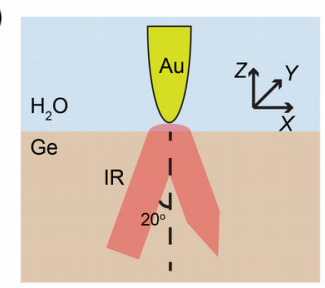

(b)

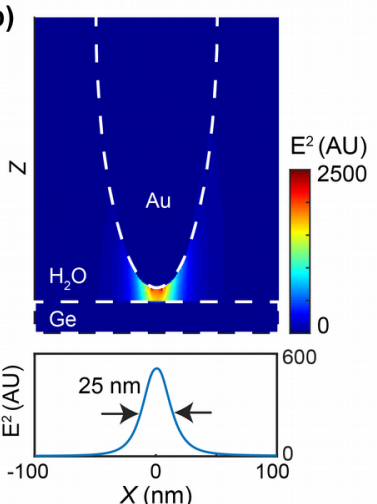

(c)

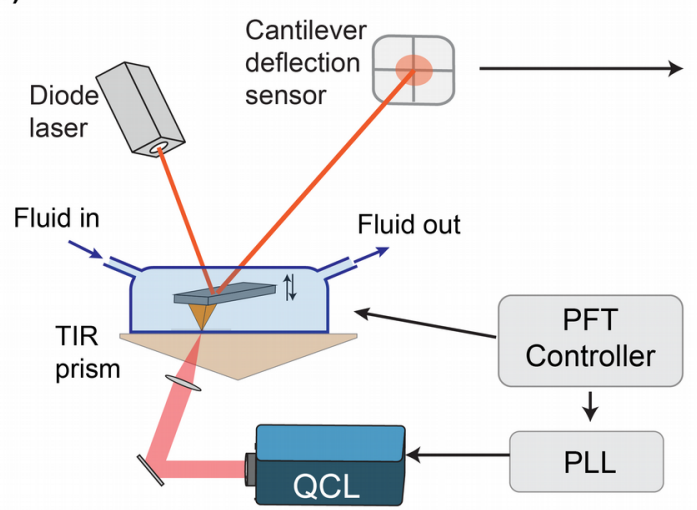

(d)
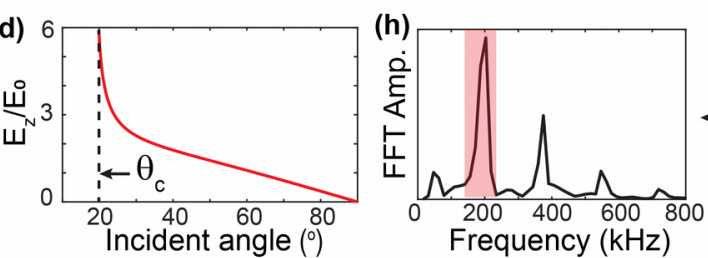
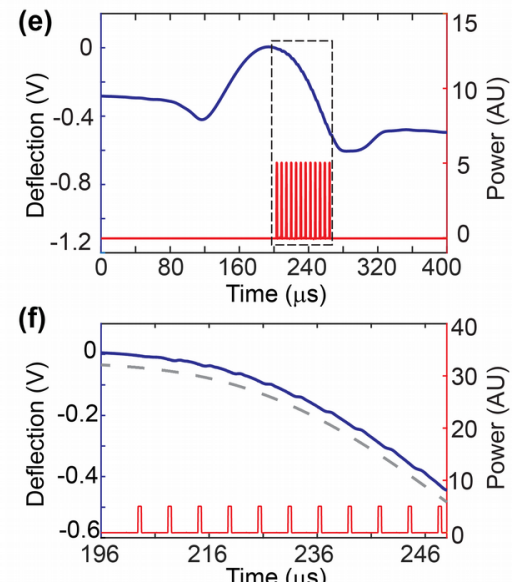

(g)

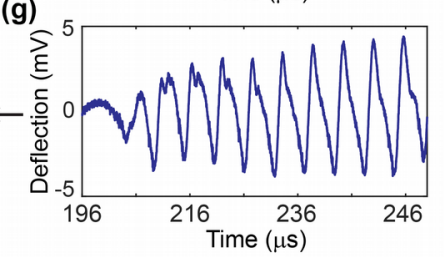

Figure 1. Design and operational principle of the LiPFIR microscopy. (a) Scheme for total internal reflection. A gold tip with an end radius of $30 \mathrm{~nm}$ is placed $3 \mathrm{~nm}$ above the Ge surface, and a p-polarized plane wave with an incident angle of $20^{\circ}$ is used to generate the evanescent field from the total internal reflection. (b) FDTD simulation of the tip-enhanced evanescent field at the $\mathrm{H}_{2} \mathrm{O} / \mathrm{Ge}$ interface. The lower panel shows the field intensity across the Ge surface. (c) Apparatus of LiPFIR microscope. (d) The relative field strength of the evanescent infrared field at the Ge/water interface at different incident angles. The highest field strength is achieved at the critical angle, located at $19^{\circ}$. (e) The cantilever deflection signal of PFT in water (blue). The infrared pulse train (red) is synchronized to every PFT cycle. (f) Zoomed-in region of laserinduced cantilever oscillations (blue) and infrared pulse train (red). A polynomial fit is performed on the cantilever deflection to obtain a smooth background (gray dashed curve, offset from the original position for the comparison). (g) High-frequency oscillations of the cantilever. Subtracting the background from the cantilever deflection signal isolates high-frequency oscillations due to the laser-induced photothermal expansion. (h) Fast Fourier transform of (g). Usually, the strongest peak in FFT is integrated and used as the LiPFIR signal. 
(a)

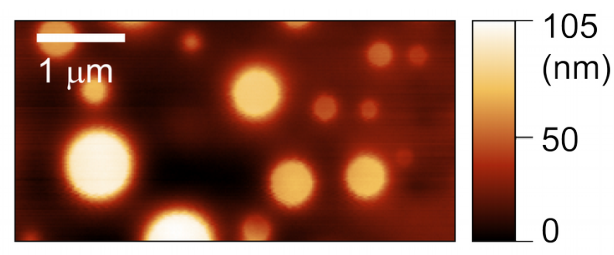

(b)

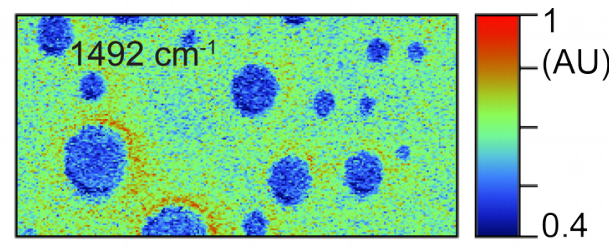

(c)

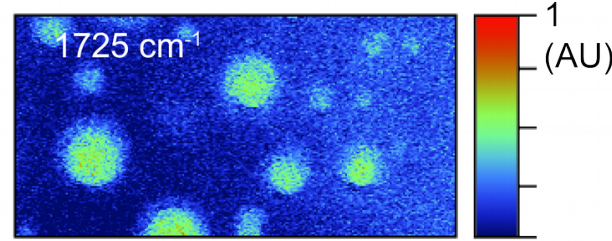

(d)

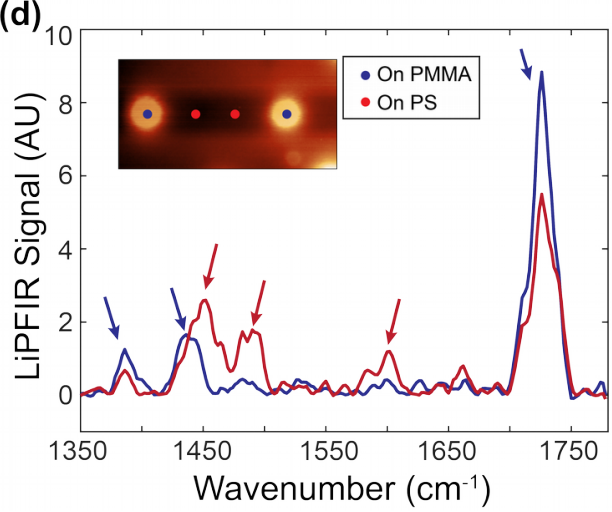

Figure 2. LiPFIR imaging and spectroscopy on PS:PMMA blend films. (a) AFM topography of a film of PS:PMMA blend in heavy water. (b-c) LiPFIR images at $1492 \mathrm{~cm}^{-1}$ (PS absorption) and $1725 \mathrm{~cm}^{-1}$ (PMMA absorption). (d) LiPFIR spectra collected from multiple locations on the PS:PMMA blend film. Inset shows the topography of a $3 \times 1.5 \mu^{2}$ area, where measuring locations are labeled as point markers. Two measurements on each domain were made using the QCL increment of $2 \mathrm{~cm}^{-1}$ and averaged spectra are shown. Spectra are smoothed using third order Savitzky-Golay method with the span of 5 . The baseline is removed. Characteristic PS and PMMA absorption peaks are indicated by red and blue arrows, respectively. 


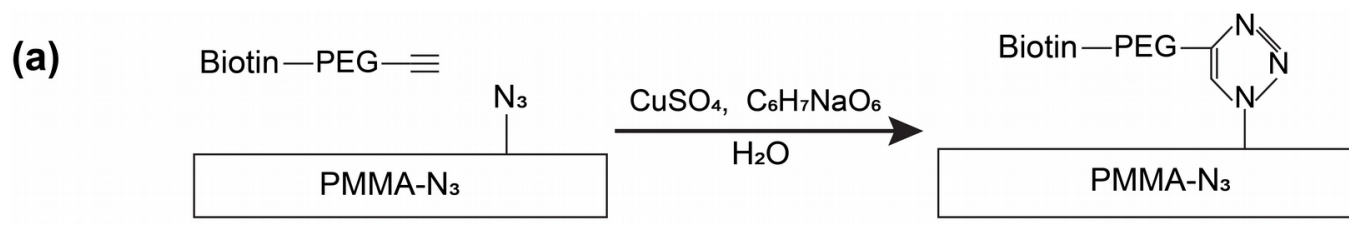

(b)
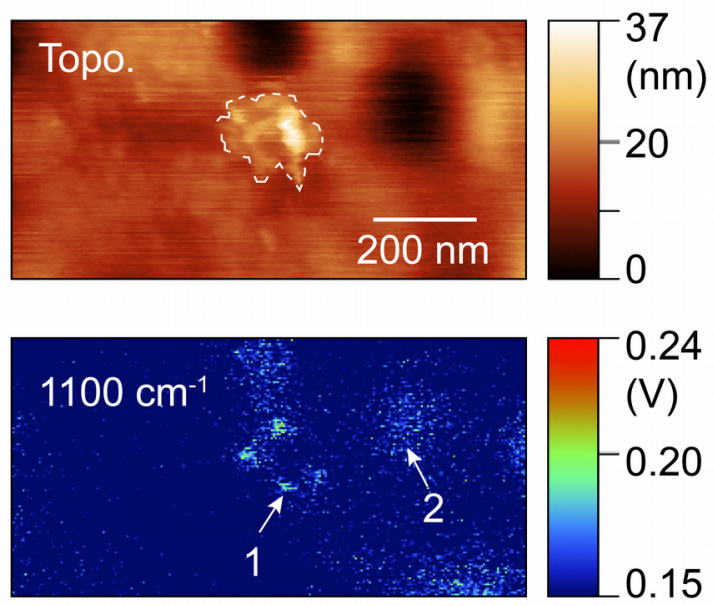

(f)

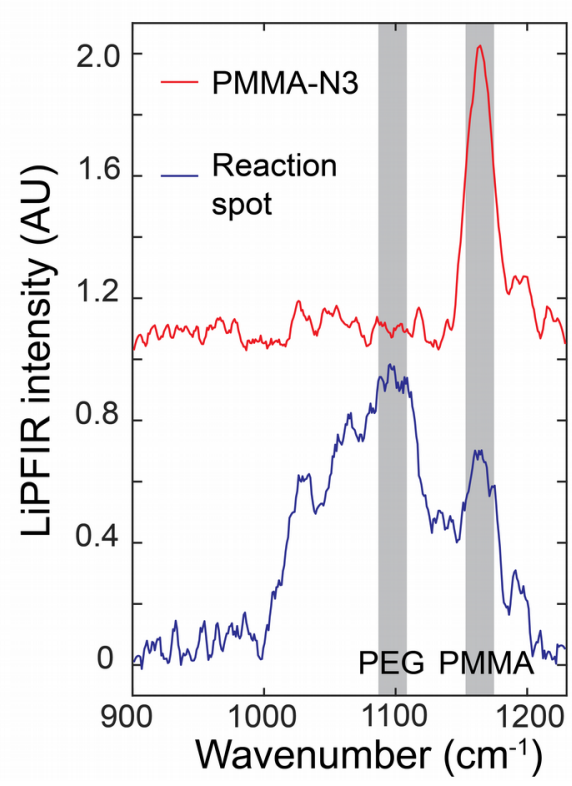

(c)

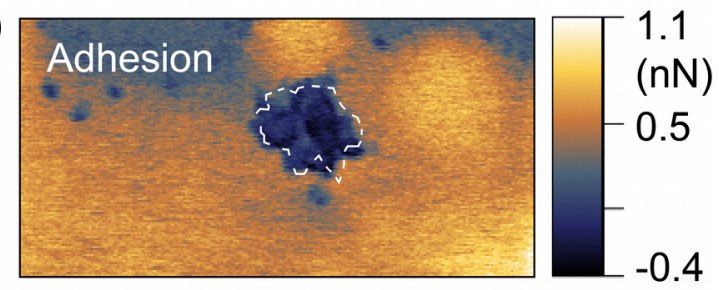

(e)

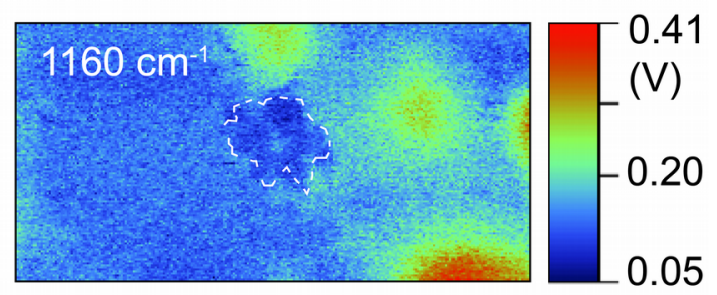

(g)

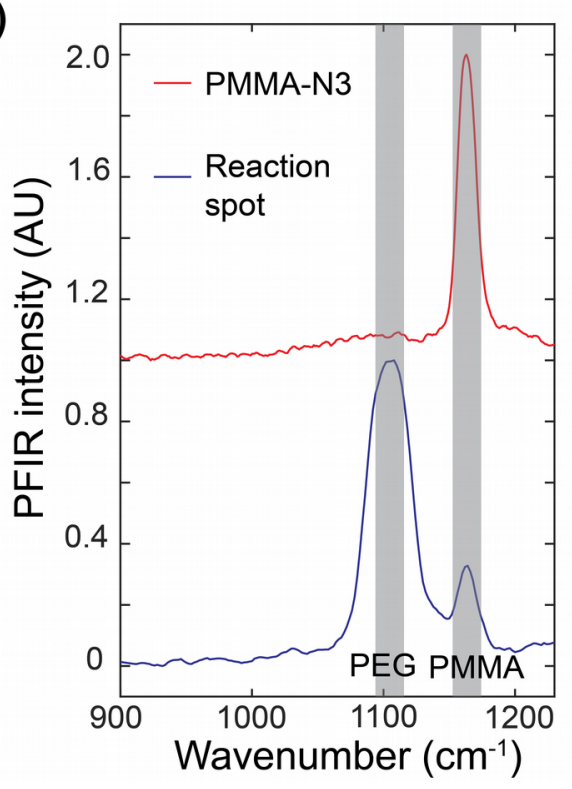

Figure 3. Revealing the click reaction site by LiPFIR. (a) Schematic of the click reaction between the alkyne-PEG-biotin in solution and the PMMA-N3 film on the germanium prism. (b) Topography of the reaction site on the PMMA-N3 film. A protruded region can be observed and is enclosed by white dashed lines. (c) Adhesion map of the same area. (d-e) LiPFIR images at $1100 \mathrm{~cm}^{-1}$ and $1160 \mathrm{~cm}^{-1}$, respectively. (f) Point spectra measured from the reaction spot (blue, position 1 in d) and the PMMA-N3 domain (red, position 2 in d). The spectra are leveled to remove the baseline. (g) Point spectra measured from the reaction spot and the PMMA-N3 
domain in the air. The wavenumber increment used in spectra measurement is $1 \mathrm{~cm}^{-1}$. The spectra are not leveled. In (f) and (g), three spectra are measured for each spot and the averaged results are smoothed using second order Savitzky-Golay method with the span of 11.

\section{(a)}
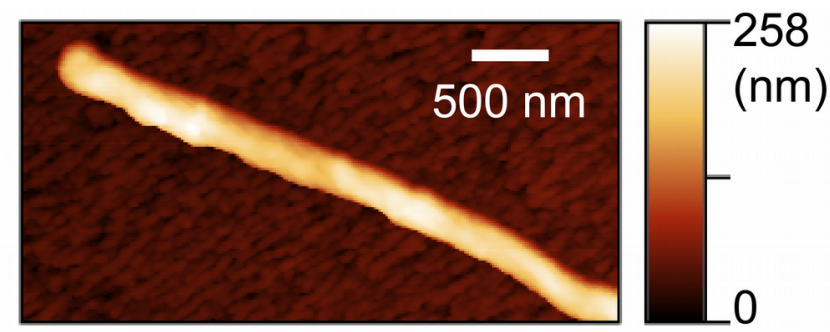

(b)
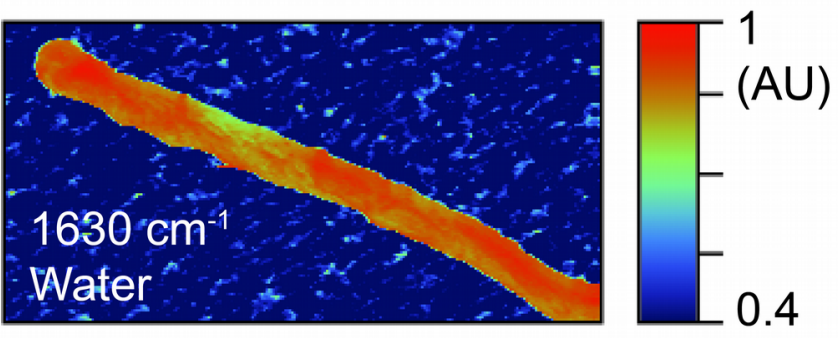

(c)

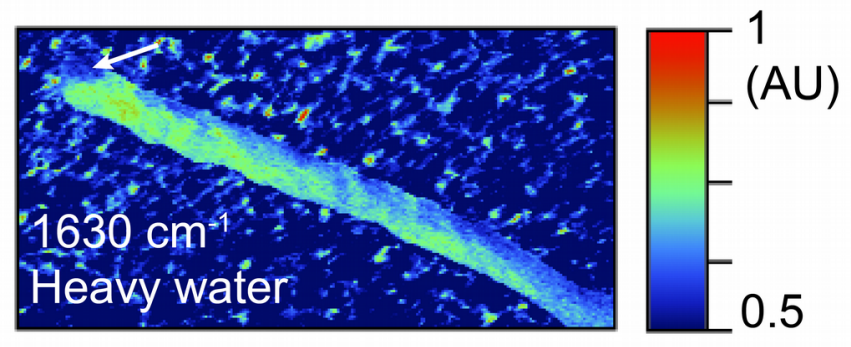

(d)
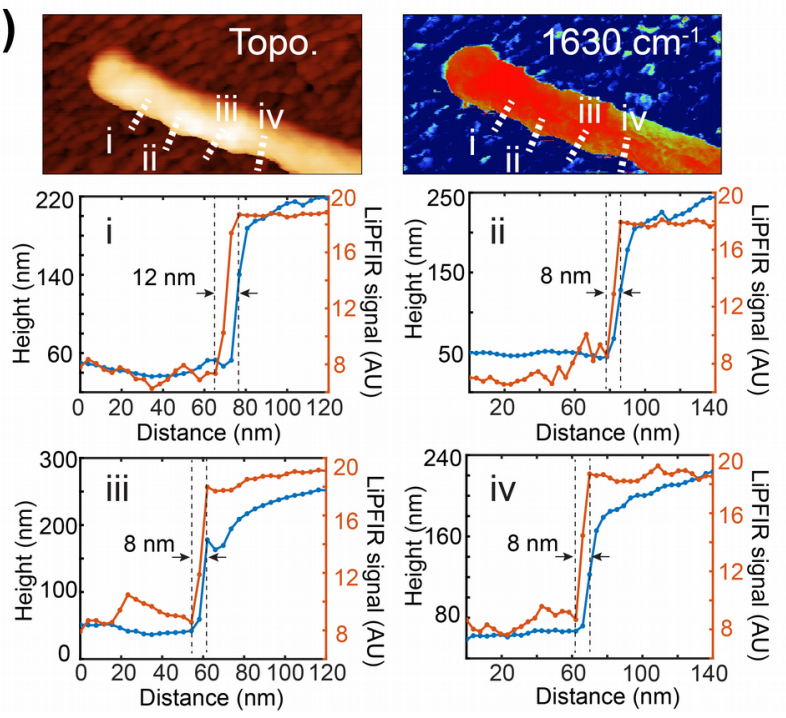

Figure 4. LiPFIR imaging of a BSA fibril in water and heavy water. (a) Topography of a 150$\mathrm{nm}$ high and 350-nm wide BSA fibril. (b) Infrared images of the BSA fibril in aqueous $0.1 \times$ 
PBS solution at $1630 \mathrm{~cm}^{-1}$. (c) Infrared images of the same BSA fibril in heavy water at $1630 \mathrm{~cm}^{-}$ ${ }^{1}$.(d) Height (blue curve) and LiPFIR signal (red curve) profiles from high-resolution topography and $1630 \mathrm{~cm}^{-1}$ infrared image of the BSA fibril in water. The pixel resolution is $3.9 \mathrm{~nm}$. Four locations (i, ii, iii and iv) of the signal extraction are labeled as white dashed lines in the topography and infrared images. Spatial resolutions of 8-12 $\mathrm{nm}$ are estimated from the lateral distance between the signal maximum and minimum across the sharp edge of the fibril.
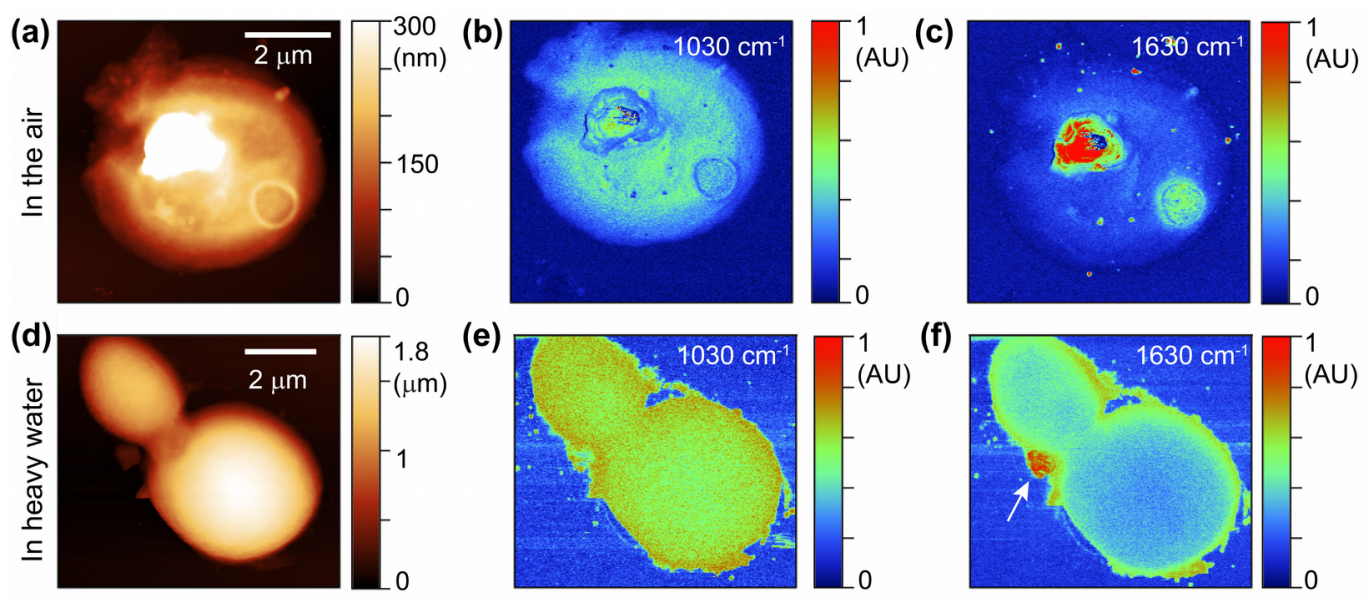

(g)
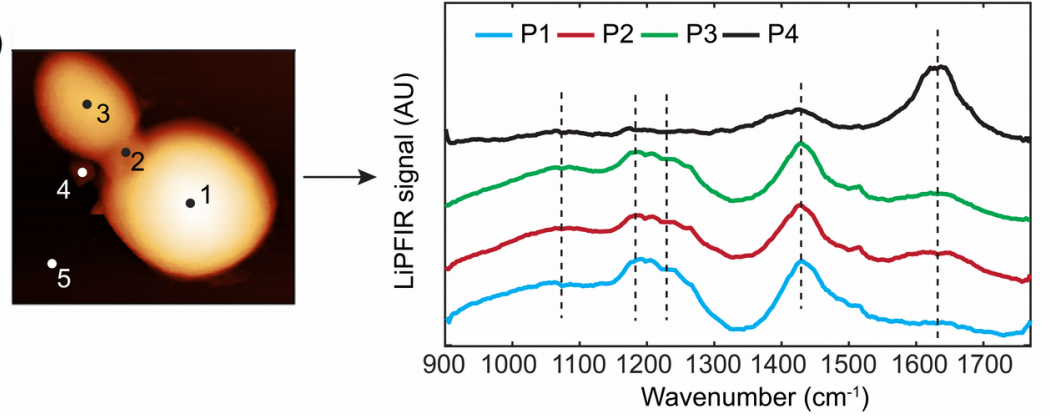

Figure 5. LiPFIR imaging and spectroscopy on zymosan particles. (a) Topography of a zymosan particle in the air. The scale bar is cut off at $300 \mathrm{~nm}$ to provide better image contrast for the bud scar of low height. (b-c) Standard PFIR images of the zymosan particle in the air at 1030 $\mathrm{cm}^{-1}$ and $1630 \mathrm{~cm}^{-1}$, respectively. (d) Topography of two zymosan particles in heavy water. (e-f) LiPFIR images of two zymosan particles in heavy water at $1030 \mathrm{~cm}^{-1}$ and $1630 \mathrm{~cm}^{-1}$, respectively. (g) The left panel indicates four spots of measurement for point spectra (positions 1 to 4). Position 5 is used to collect the baseline. The right panel shows collected spectra from four 
different locations on the two zymosan particles. Peaks at 1080, 1190, 1220, 1430 and $1630 \mathrm{~cm}^{-1}$ are labeled with dashed lines. 